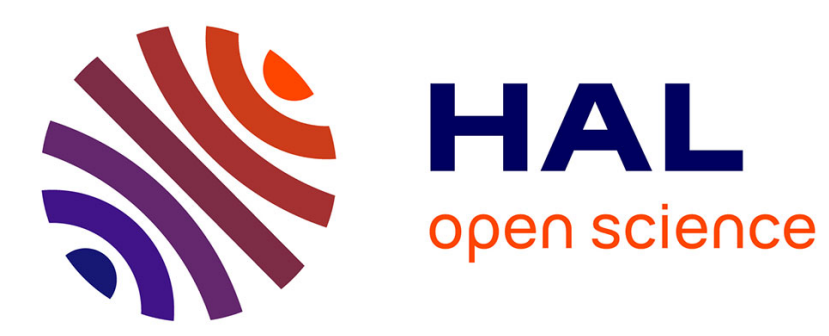

\title{
Joyman: a Human-Scale Joystick for Navigating in Virtual Worlds
}

\author{
Maud Marchal, Julien Pettré, Anatole Lécuyer
}

\section{To cite this version:}

Maud Marchal, Julien Pettré, Anatole Lécuyer. Joyman: a Human-Scale Joystick for Navigating in Virtual Worlds. IEEE Symposium on 3D User Interfaces 2011 (3DUI), Mar 2011, Singapour, Singapore. inria-00567437

\section{HAL Id: inria-00567437 https://hal.inria.fr/inria-00567437}

Submitted on 21 Feb 2011

HAL is a multi-disciplinary open access archive for the deposit and dissemination of scientific research documents, whether they are published or not. The documents may come from teaching and research institutions in France or abroad, or from public or private research centers.
L'archive ouverte pluridisciplinaire HAL, est destinée au dépôt et à la diffusion de documents scientifiques de niveau recherche, publiés ou non, émanant des établissements d'enseignement et de recherche français ou étrangers, des laboratoires publics ou privés. 


\section{Joyman: a Human-Scale Joystick for Navigating in Virtual Worlds}

\author{
Maud Marchal* \\ INSA/INRIA Rennes, France
}

\author{
Julien Pettré * \\ INRIA Rennes, France
}

\author{
Anatole Lécuyer * \\ INRIA Rennes, France
}

\begin{abstract}
In this paper, we propose a novel interface called Joyman, designed for immersive locomotion in virtual environments. Whereas many previous interfaces preserve or stimulate the users proprioception, the Joyman aims at preserving equilibrioception in order to improve the feeling of immersion during virtual locomotion tasks. The proposed interface is based on the metaphor of a human-scale joystick. The device has a simple mechanical design that allows a user to indicate his virtual navigation intentions by leaning accordingly. We also propose a control law inspired by the biomechanics of the human locomotion to transform the measured leaning angle into a walking direction and speed - i.e., a virtual velocity vector. A preliminary evaluation was conducted in order to evaluate the advantages and drawbacks of the proposed interface and to better draw the future expectations of such a device.
\end{abstract}

Index Terms: H.5.1 [INFORMATION INTERFACES AND PRESENTATION]: Multimedia Information Systems-Artificial, augmented, and virtual realities; H.5.2 [INFORMATION INTERFACES AND PRESENTATION]: User Interfaces-Input devices and strategies

\section{INTRODUCTION}

Navigation is one of the fundamental tasks needed for 3D interaction with Virtual Environments [3]. The possibility to walk inside the virtual environment (VE) is necessary in many applications of Virtual Reality (VR) such as for virtual visits (review of architectural and urban projects), training tasks or videogames. However, it is still a challenge to allow a user endlessly walking a virtual world in an immersive manner when the physical real workspace is limited. The difference between the dimensions of the real and virtual worlds prevent from tracking users and directly transposing the walking motion into the virtual world.

In order to provide VR users with realistic sensations of walking while keeping them in their limited workspace, numerous types of VR interfaces have been proposed so far. Thus, several types of peripheral devices have been proposed. Simple manual devices such as keyboards, mices or joysticks are widely available. They allow users to indicate their locomotion wills into the virtual world but only involve the users' hands and arms whereas real locomotion involves all of the human limbs. Thus, they cannot be satisfactory used in the frame of immersive applications. Locomotion interfaces made of sophisticated treadmills and tracking systems have been designed to improve the walking sensations in VR $[8,15]$. They enable users to endlessly walk while their global displacement is mechanically compensated. Such systems remain however highly expensive and cumbersome and necessarily distort the vestibular sensory feedback - i.e., equilibrioception - due to motion compensation. Finally, software solutions have been proposed to enhance the sensations of walking in VR. Some techniques [11, 12,4] allows the user to really walk in the real workspace without reaching its limits but do not prevent break of immersion or need a big real workspace.

*e-mail: \{maud.marchal,julien.pettre,anatole.lecuyer\}@inria.fr
Other techniques such as the Walking-In-Place paradigm [14] preserve both the immersion and the real somatosensory feedback as users virtually walk by performing real walking motions. But the absence of real global displacement neglect equilibrioception for the benefit of proprioception.

In this paper, we explore the use of a novel interface for navigating into virtual worlds that tends to preserve equilibrioception in place of proprioception. It is often said that walking is constantly falling ahead. It is also known that head trajectory precedes the global locomotion trajectory. This justifies our work direction towards the design of a device involving the vestibular system that stands in the human head. Our second objective is to study affordable devices and propose a new interface that does not involve either active mechanical devices or complex tracking systems. The concept of our new interface, called Joyman, is illustrated in Figure 1. The proposed interface is based on the metaphor of a human-scale joystick. The device has a simple mechanical design that allows a user to indicate his virtual navigation intentions by leaning accordingly.
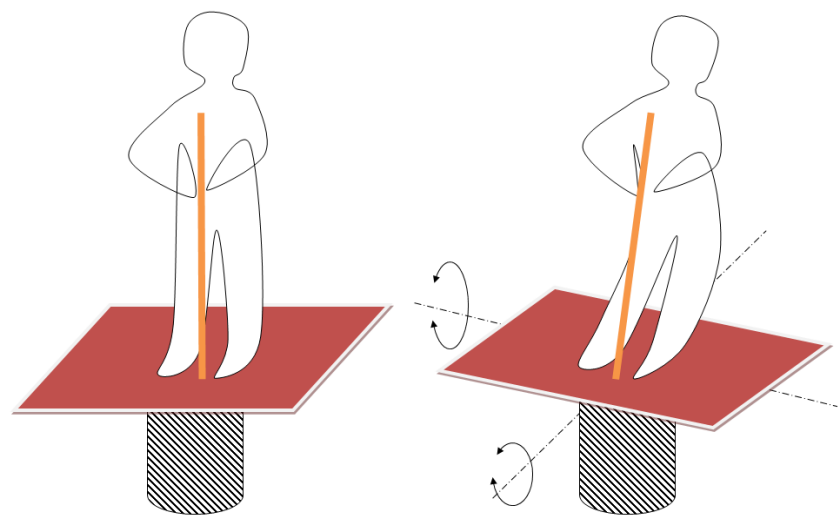

Figure 1: Principle of the Joyman interface for walking into virtual worlds. Following the example of joystick and standing on an articulated platform, users indicates their desired walking direction by leaning.

The main contributions of our paper are the following:

- A novel and original interface involving equilibrioception to enhance immersion in virtual environments when performing locomotion tasks. The new interface is based on a mechanical design that meets the requirements of the limited real workspace while preserving the locomotion sensations. It does not involve either active mechanical devices or complex tracking systems.

- A combination of a new peripheral with a control law inspired by the non-holonomic nature of the human walk. The law transforms the device orientation into locomotion velocities in order to compose a complete locomotion interface. This law express attainable turning velocities as a function of the tangential one: it has been observed that the faster the walk the lower the attainable turning speed. 
The remaining of the paper is organized as follows: Section 2 provides an overview of existing interfaces and discuss their advantages and drawbacks. In Section 3, we introduce the description of our novel locomotion interface. We also present the associated control law. Finally, Section 4 details the preliminary experimental evaluation of our locomotion interface before ending with discussion and perspectives for the Joyman interface.

\section{Related Work}

In order to provide VR users with realistic sensations of locomotion while keeping them in their limited workspace and without any break in the immersion, numerous types of VR interfaces have been proposed so far. The most straightforward solution probably consists in walking naturally in the real world, using for instance Head Mounted Display (HMD) together with a tracking system which measures head position for updating virtual camera position and orientation in the virtual environment. However, this solution can rarely be used due to the limited physical workspace in the real world, as well as limitations of the tracking devices.

To overcome this problem, numerous "locomotion interfaces" have been designed. The locomotion interfaces keep the user within the limits of the physical workspace while allowing to navigate infinitely in the virtual world. A classical hardware approach consists in using a treadmill which can be either unidirectional (such as the Sarcos Treadport [15]) or omni-directional (such as the Torus Treadmill [8]). Other locomotion interfaces can be based on feetplatforms [7], walking inside a rotating sphere [5] or a set of moving tiles [6], standing on a Cybercarpet [13]. These locomotion interfaces have the advantage of providing realistic walking sensations, involving proprioceptive information. However, the current locomotion interfaces are often too expensive, cumbersome, or complex for being integrated easily in both VR applications.

Besides, classical input devices such as mice, keyboards and joysticks can also be used within interaction metaphors to navigate from one virtual place to another [3]. However, such interaction techniques, relying exclusively on manipulated input devices, do not provide any proprioceptive or vestibular feedback of walking and lead to a poor sensation of locomotion in the VE.

Thus, numerous interaction techniques have been developed to provide software-based navigation capabilities in VR. Software solutions have been proposed in order to keep the user inside the real workspace limits while he is really wallking, such as the World in Miniature technique [11], the Magic Barrier Tape [4] or the FreezeBackup technique [18]. However these techniques induce breaks in the feeling of immersion during the navigation in a virtual environment. Redirected Walking Technique [12] or Motion Compression [10] preserve the immersion by forcing the user into walking in a curved path in the real world while walking in a straight line in the virtual world. However, these techniques require large workspaces, can be confusing when doing unpredictable or quick changes of direction, and may require distracting events.

Other solutions have been proposed to enhance the sensation of walking in virtual worlds without modifying the user position in the real environment. An efficient solution consists in playing with visual feedback and adding artificial camera motions [9]. The Walking-In-Place technique has been introduced by Slater et al. [14] to enable a real physical walking movement and an efficient navigation technique in $3 \mathrm{D}$ virtual environments. In this interaction paradigm, the user is walking in place in the real world, allowing proprioceptive feedback while keeping him in the real workspace. However, the absence of real global displacement neglects equilibrioception.

In this paper, we would like to explore the possibility of an interface that aims at preserving equilibrioception in place of proprioception. The ChairIO project [2] also implies equilibrioception but the user is seated while he is navigating in the virtual world, de- creasing the immersion, especially for locomotion tasks. Our new interface is based on the metaphor of a human-scale joystick. Some devices have already been built [17] but they are not designed for enhancing immersion in VE, especially as they are not combined with a control law allowing to reproduce locomotion tasks. The main principles and the design of our new interface, called Joyman, are detailed in the next section.

\section{DEscription OF the JoYman INTERFACE}

The Joyman is a new interface to navigate into virtual worlds. One main objective of this interface is to go towards realistic locomotion trajectories in the virtual environment. The Joyman meets this objective by combining two components: a peripheral device allowing users to indicate the desired direction of locomotion and a control law which transforms the device state into a virtual velocity vector. The mechanical design of the device is based on the metaphor of a human-scale joystick and mainly consists of a board upon which the user stands. The inclination of this board can be changed by the user. Thus, the user indicates the desired locomotion direction by tilting the platform in the corresponding direction. The mechanical design of the platform allows the user to change the platform inclination by leaning and prevents him from falling, whereas repealing forces tend to maintain the platform horizontal. The control law transforms the device state (pitch and roll angles) into a virtual locomotion velocity vector. The proposed law ensures that humanly feasible velocities are achieved during virtual locomotion. Particularly, the tangential velocity is bounded and depends on the desired angular velocity. These two components are detailed below.

\subsection{Device Mechanical Design}

The proposed mechanical design of the interface is proposed in Figure 2 .

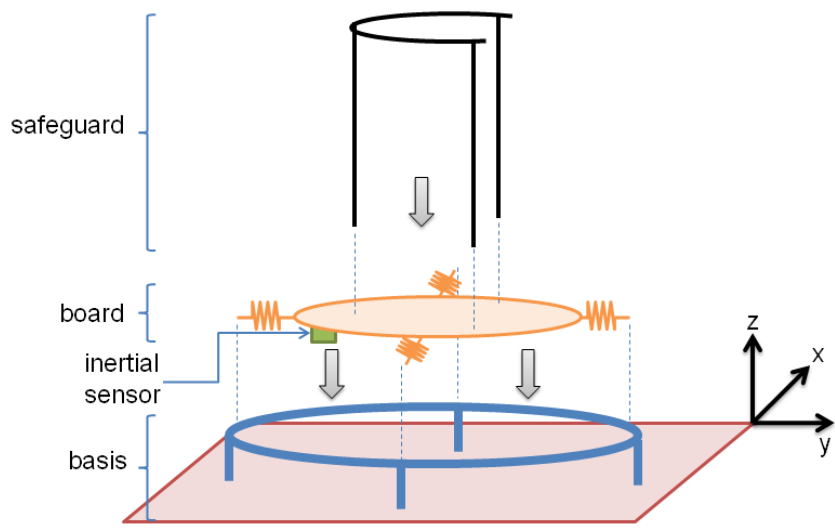

Figure 2: Schematic representation of the main elements composing our peripheral device. The board and the basis are articulated using springs. Springs generate repealing forces that tend to maintain the board in the horizontal plane, however, the board can be freely oriented around the two horizontal axis (pitch and roll) with limited range. Users act on the device by standing on it, and apply forces in order to tilt the board by leaning. The board inclination is measured using an inertial sensor. The safeguard prevents users from falling when leaning.

The key principle of the Joyman is to let the user indicate the desired direction of his virtual locomotion by leaning in the corresponding direction. In other words, our objective is to let a user control his locomotion from his equilibrioception. This main functionality stressed at first the mechanical design of the Joyman device. 
The main properties of our design are :

- the user should be able to stand on the platform, which is the natural position in the task of locomotion;

- the user should feel perfectly safe when using the platform which, as a result, should prevent him from falling;

- the user should be able to lean over the limit of his own balance in order to increase the amplitude of vestibular sensations;

- the device should tend to bring the user back in vertical position to avoid highly tiring manœuvers during its use.

We also carefully considered other criteria during the device design process: the most important was to build an affordable interface. Exploring the equilibrioception in place of proprioception to control oneself locomotion in the virtual world is a promising direction to lower these costs. However, we did not want to lower the costs by degrading the feeling of immersion provided by the interface. We aim at building a new interface that does not involve any active mechanical device in its movement and does not imply the use of sophisticated and complex tracking devices. We meet the objectives listed above based on a simple mechanical device as illustrated in Figure 2.

The device is made of 4 components:

The basis supports the whole device. It is made of a flat square platform lying on the ground and of a circle shaped steel frame linked to the board by legs. The legs and the platform are fixed together and the board is large enough to prevent the device from tipping over.

The board is the element supporting users. It is circle shaped and linked to the frame by springs. As a result, the board is free relatively to the frame within limited range of motion. We can approximately but reasonably consider that the board is articulated to the frame by two degrees of freedom which are rotations around the $x$ and $y$ axis as illustrated in Figure 2 .

The safeguard is rigidly fixed to the board and prevent users from falling.

The inertial sensor is rigidly attached to the board and acquires the current orientation of the board.

Figure 3 displays the peripheral device built in our lab following the simple mechanical design described above.
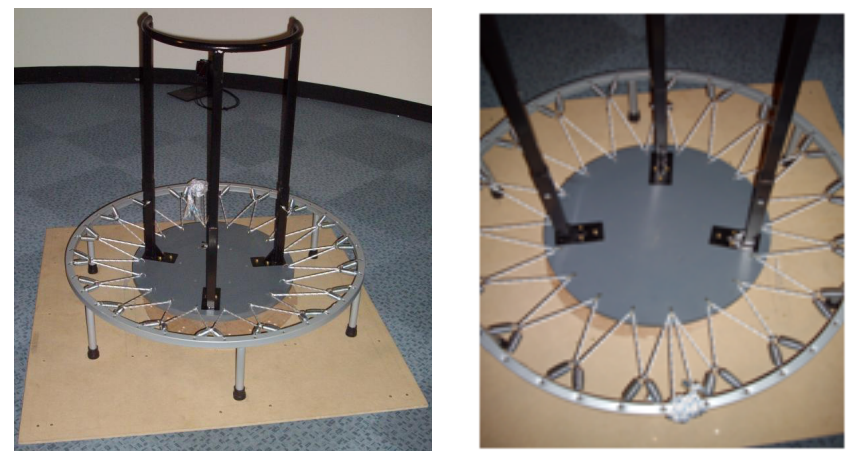

Figure 3: Illustration of the new peripheral device.

The basis is made of a square woodcut platform. The steel frame is the one of a trampoline where the springs have also been retrieved
(1 $\mathrm{m}$ diameter). The basis is a machined plastic plate $(0.55 \mathrm{~m}$ diameter) equipped with 18 hooks on its rim. A rigid rope is tied up between the hooks and the springs attach the board to the frame. Finally, the safeguard is made of welded iron tubes. Its height can be adapted to each user (height between $0.8 \mathrm{~m}$ and $1.2 \mathrm{~m}$ ). Building costs did not exceed $500 \$$ in spite of the fact that this device is a unique prototype excluding the inertial sensor.

Illustrations of the peripheral device in use are provided in Figure 4. The user can stand straight on the platform and has to lean in any direction to start the locomotion in the virtual world. The control law that allows to navigate in a VE with the new interface is described in the next paragraph.
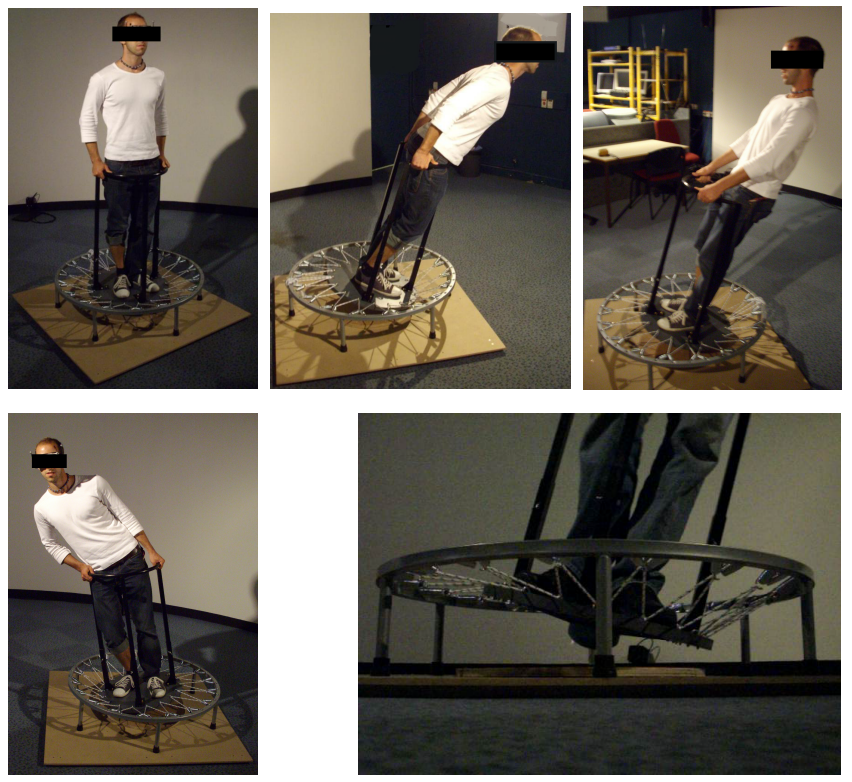

Figure 4: Illustration of the peripheral device in use. Users can stand straight on the board, repealing forces make easy keeping the platform inclination. Users have to lean to start locomotion in virtual worlds. All the possible leaning directions are displayed.

\subsection{Virtual Locomotion Control}

The main steps of the virtual locomotion control are summarized in Figure 5. The control is composed of two elements detailed in the following paragraph: a locomotion model and a control law.

\subsubsection{Locomotion model}

The locomotion model allows to translate the position and orientation of the platform into a virtual motion in the VE. We exploited experimental observations of the walking human trajectory to build our model [1].

We model the position $P$ of the user in the virtual space as an oriented point moving in a horizontal plane (see Figure 5):

$$
P=\left[\begin{array}{l}
x \\
y \\
\theta
\end{array}\right]
$$

The virtual motion is velocity controlled. We assume the virtual trajectory is non-holonomic, which means that the velocity vector orientation and the body orientation are always identical. The nonholonomic nature of walking human trajectory has been experimentally observed in [1]. 


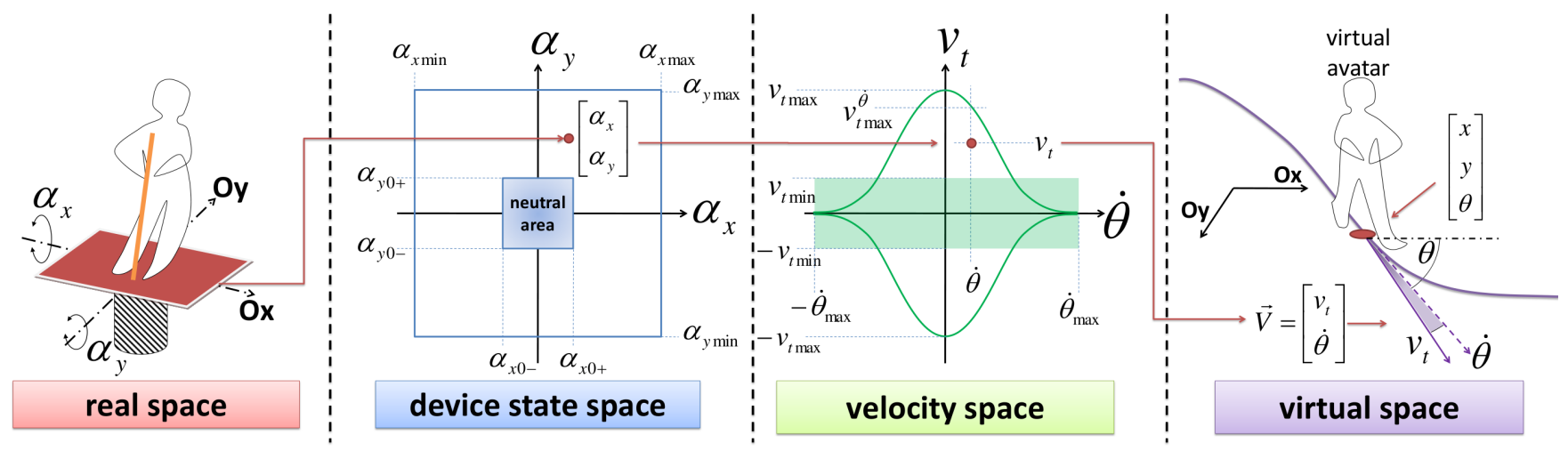

Figure 5: Summary of the different steps for the virtual locomotion control. From the position and orientation of the Joyman, we can compute the virtual velocity vector by using our locomotion model and control law.

Non-holonomy constraint allows us to decompose the velocity vector $V$ as follows:

$$
V=v_{t} \cdot\left[\begin{array}{c}
\cos (\theta) \\
\sin (\theta)
\end{array}\right]
$$

Such a decomposition allows us to independently control the tangential speed $v_{t}$ and the orientation $\theta$. Human tangential velocity is limited. We denote $v_{t_{\max }}$ the maximum tangential velocity bound. By default, we set this bound at $v_{t_{\max }}=1.4 \mathrm{~m} . \mathrm{s}^{-1}$. It has equally been observed that very slow walking velocities are never reached: such velocities are humanly feasible but not used in practice. We thus define $v_{t_{\min }}=0.6 \mathrm{~m} . \mathrm{s}^{-1}$. It has been however observed that changing orientation $\theta$ affects the amplitude of $v_{t}$ during human locomotion [19]: humans decelerate when turning. In order to take into account such an observation, we define $v_{t_{\max }}^{\dot{\theta}}$ the bound of the tangential velocity knowing the current turning velocity $\dot{\theta} \cdot v_{t_{\max }}^{\dot{\theta}}$ is defined as follows:

$$
\begin{aligned}
v_{t_{\text {max }}}^{\dot{\theta}} & =a \cdot v_{t_{\text {max }}} \cdot e^{-b \cdot \frac{\dot{\theta}}{c}} \\
v_{t_{\text {min }}}^{\dot{\theta}} & =a \cdot v_{t_{\text {min }}} \cdot e^{-b \cdot \frac{\dot{\theta}}{c}}
\end{aligned}
$$

where $a, b$ and $c$ are parameters. As a result, we model the reachable tangential velocity $v_{t_{\max }}^{\dot{\theta}}$ as a Gaussian function of the current turning velocity $\dot{\theta}$. The higher $\dot{\theta}$, the lower the tangential velocity bound. By default, we arbitrarily choose: $a=1.07, b=0.5$ and $c=0.7$. Such values match the experimental observations provided in [19]. Finally, the absolute value of the angular velocity is also bounded to $\dot{\theta}_{\max }$. We arbitrarily choose $\dot{\theta}_{\max }=1 \mathrm{rad} . \mathrm{s}^{-1}$.

\subsubsection{Control Law}

The control law allows users to modify the virtual velocity vector $V$ by standing on the device and leaning. The modification of the platform orientation affects the state of the device $s_{d} . s_{d}$ is defined by the orientation of the board $\alpha_{x}$ and $\alpha_{y}$ relatively to the two horizontal axis $\overrightarrow{O x}$ and $\overrightarrow{O y}$, as measured by the inertial sensor:

$$
s_{d}=\left[\begin{array}{l}
\alpha_{x} \\
\alpha_{y}
\end{array}\right]
$$

We neglect the orientation around the $O z$ axis. During calibration stage, we ask the user to stand on the platform and to successively firmly lean towards all the cardinal directions. We estimate the reachable bounds of the board orientation by averaging the reached orientation over a short period of time, $\alpha_{x_{\min }}, \alpha_{x_{\max }}, \alpha_{y_{\min }}$ and $\alpha_{y_{\max }}$.
We also ask the user to stand straight on the platform and define a neutral area bounded by $\alpha_{x_{0+}}, \alpha_{x_{0-}}, \alpha_{y_{0+}}$ and $\alpha_{y_{0-}}$.

We want the user to control his tangential velocity $v_{t}$ by leaning in forward or backward direction, i.e., by playing on $\alpha_{x}$, whilst angular velocity $\dot{\theta}$ is controlled by leaning on the sides, i.e., by playing on the $\alpha_{y}$ value. The angular velocity is controlled as follows:

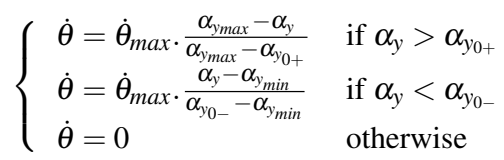

which allows to deduce $v_{t_{\max }}^{\dot{\theta}}$ and $v_{t_{\min }}^{\dot{\theta}}$ according to equation 3 and finally $v_{t}$ :

$$
\begin{cases}v_{t}=v_{t_{\min }}^{\dot{\theta}}+\left(v_{t_{\max }}^{\dot{\theta}}-v_{t_{\min }}^{\dot{\theta}}\right) \cdot \frac{\alpha_{x_{\max }-\alpha_{x}}}{\alpha_{x_{\max }-\alpha_{x_{0+}}}} & \text { if } \alpha_{x}>\alpha_{x_{0+}} \\ v_{t}=-v_{t_{\min }}^{\dot{\theta}}+\left(v_{t_{\max }}^{\dot{\theta}}-v_{t_{\min }}^{\dot{\theta}}\right) \cdot \frac{\alpha_{x}-\alpha_{x_{\min }}}{\alpha_{x_{0-}}-\alpha_{x_{\min }}} & \text { if } \alpha_{x}<\alpha_{x_{0-}} \\ v_{t}=0 & \text { otherwise }\end{cases}
$$

At each time step $n$, the virtual velocity vector $V_{n}=\left[v_{t_{n}}, \dot{\theta}_{n}\right]$ is thus deduced from $s_{d}$, the state of the device. Before updating the simulation accordingly, we check that no unbelievable acceleration is performed. Thus, given the previous velocity vector $V_{n-1}$, we finally compute the effective velocity vector $V_{f}$ :

$$
V_{f}=V_{n-1}+\left\lfloor\frac{V_{n}-V_{n-1}}{\Delta t}\right\rceil
$$

where $\Delta t$ the simulation time step, and \lfloor\rceil a function that truncates the velocity vector variation so that the absolute tangential acceleration does not exceed $1 \mathrm{~m} \cdot \mathrm{s}^{-2}$ and the angular one does not exceed $1 \mathrm{rad} . \mathrm{s}^{-2}$.

\subsection{Summary of Joyman Interface}

The Joyman interface is designed for immersive virtual locomotion into virtual worlds. Our main aim was to explore the possibility of an interface that tends to preserve the equilibrioception in place of proprioception, in contrary to many current interfaces. The mechanical design of our platform is relatively simple. We showed how the proposed device allows a user to safely perform exaggerated leaning motion, over the limit of balance, in order to indicate his virtual navigation wills. We here remind our long-term objective to be able to perform realistic locomotion trajectories into the virtual world. What can we expect from the Joyman interface? 
Immersion The device does not limit technical choices about visual or audio feedback. Typically, the device can be directly used in any immersive environment. More important, the initiation of the locomotion is made by leaning ahead. This corresponds to the real motion to initiate walking, which is considered to be a constant loose of balance and succession of falling ahead. We expect this interaction mode, where vestibular sensory system stimulation is preserved, to be greatly immersive.

Realism We expect that the dynamics of the required manœuvers to operate the Joyman are close to the ones of real walking motions. As an example, using the Joyman, users perform a left turn following a right turn by changing the platform orientation toward its opposite using their whole body. Involved inertias and frictions prevent from performing this change of state immediately: this reproduces the walking behavior during which the inclination of the body is naturally adapted to face centrifuge force. We clearly do not expect users to achieve efficient navigation (in terms of task completion time) using the Joyman.

\section{Preliminary Evaluation}

As a preliminary evaluation, we propose to compare our interface to the joystick, a classical peripheral often used in VR applications. The joystick can be considered as one of the most performant interface to achieve efficient navigation in terms of task completion time. Thus, the first objective of our evaluation was to quantify the loss of performances of our interface compared to the joystick (our interface involves namely the whole body compared to the joystick where only hands and arms are used). The experiments were conducted using 3D VE displayed either on a screen or on a HeadMounted Display (HMD). We investigated the effectiveness of our interface to travel complex paths composed of different gates placed in the VE. The second objective of our evaluation was to verify if we can obtain a more immersive navigation with our interface compared to classical peripherals already used in VE, ie. the joystick. Thus, a subjective questionnaire was proposed to the participants to evaluate their subjective preferences in terms of quality of the VE navigation.

\subsection{Method}

\subsubsection{Virtual Environment}

The evaluation was performed within a $3 \mathrm{D}$ virtual environment without any contextual cues. The only landmarks were the gates that the user had to navigate through. A fog effect was added to mask the distant gates, allowing to perceive only the 2 or 3 closest gates. A texture on the ground provided useful visual flow information during the navigation. The scene was normally illuminated, and no shadows were drawn as illustrated in Figure 6.

\subsubsection{Sample Population}

Sixteen participants aged from 19 to $28($ Mean $=23.4, \mathrm{SD}=2.4)$ performed the experiment. One participant was left-handed and none of them had known perception disorders. All participants were naïve with respect to the proposed interface, as well as to the experimental setup and purpose of the experiment.

\subsubsection{Experimental Conditions}

The experiment was carried out using two visual conditions: HMD or a screen. With these two different visual conditions, we would like to evaluate if the display manner (fixed screen or screen moving with the body for the HMD) has an influence on the user sensations. The two visual conditions and associated experimental configurations are illustrated in Figure 7.

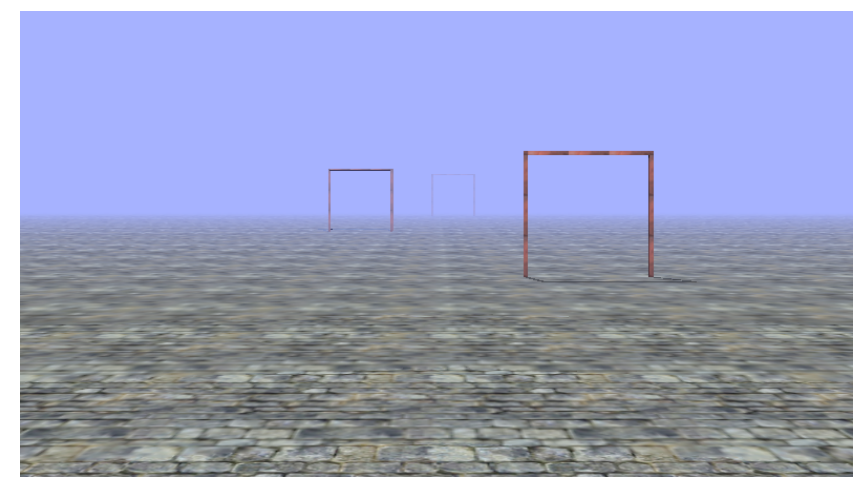

Figure 6: Snapshot of the virtual environment used during the evaluation. The different gates represent the path that the user had to navigate through.

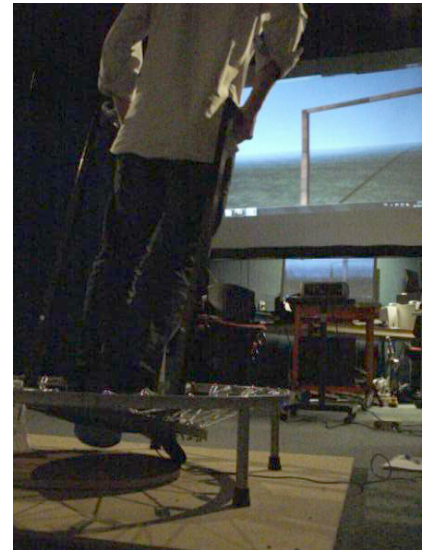

(a)

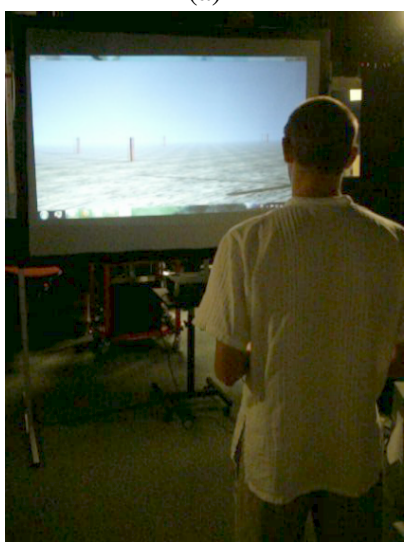

(c)

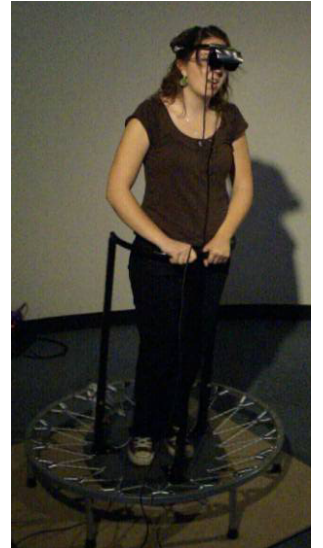

(b)

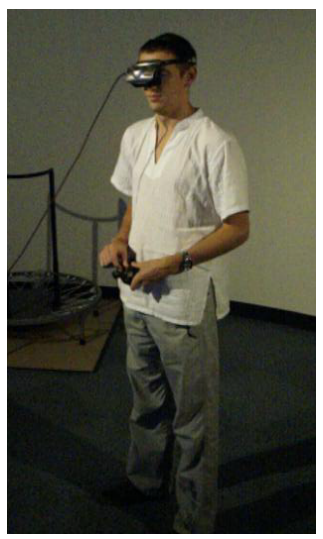

(d)
Figure 7: Description of the experimental setup for the 4 configurations: the virtual scene was visually perceived through HMD (b and d) or through a projection screen ( $a$ and $b$ ); locomotion was controlled using the Joyman ( $a$ and $b$ ) or a joystick (c and d). 
For the screen condition, the luminosity was controlled in the room using two projectors. The participants were at a distance of $2 \mathrm{~m}$ in front of a $1.72 \mathrm{~m}$ large and $1.24 \mathrm{~m}$ height back-projected screen (physical field of view of 60 degrees horizontally and 45 degrees vertically). The resulting image had a resolution of $1600 \times$ 1200 pixels. We used monoscopic rendering, with a frame rate at $60 \mathrm{~Hz}$. The projector used was a DepthQ Stereoscopic. For the HMD condition, we used the eMagin Z800 Head Mounted Display, at $60 \mathrm{~Hz}$ (physical field of view of 40 degrees diagonally). The video image was generated without using any head tracking sensor. The participants were standing either on the Joyman or on the ground to complete the task.

\subsubsection{Experimental Apparatus}

In our experiment, the goal was to compare our interface to the joystick, a classical interface device widely used in VR applications. In addition, we used two different visual conditions (HMD and a screen). During the experiment, the participants had to navigate in the VE through 2 different paths composed of 8 gates each. The gates were 3 meters by 3 meters wide and were disposed in order to form a slalom.

The participants were able to choose at the beginning of each block of conditions when they wanted to launch the experiment. At any time during the experiment, they had the possibility to make a break. After each trial, the participants were automatically teleported to the beginning of the next trial. A black screen displayed $2 \mathrm{~s}$ notified the beginning of the new trial.

\subsubsection{Experimental Plan}

The participants were exposed to 4 blocks of 8 trials each: one block for each of the possible combinations of the experimental conditions. The combinations were the following: (1) Joyman, using a HMD; (2) Joyman, using the screen; (3) Joystick, using a HMD; (4) Joystick, using the screen.

The participants were split in 4 groups equally composed of 4 people each. Two groups started with the Joyman configuration and the 2 others with the Joystick configuration. We counter-balanced the conditions between them, meaning that the group starting with the HMD condition in the Joyman configuration starts with the screen condition in the Joystick configuration. For each of the 8 trials of a block, the participants had to navigate through the 2 paths (4 for each, in a random order).

\subsection{Results}

\subsubsection{Collected Data}

In our evaluation, we measured for each participant the task completion for each trial and the percentage of success for the different gates. A subjective questionnaire was also proposed to evaluate 8 criteria.

\subsubsection{Performances: Task Completion Time and Accuracy}

For each participant, the task completion time of each trial was measured for the different experimental conditions. A repeated two-way ANOVA was performed on the two different interfaces and the two visual conditions. The ANOVA accounting for the visual conditions and the task completion time revealed a significant effect $(F(1,526)=11.63$, p-value $<0.001)$. A significant effect was also found for the interfaces and the task completion time $(F(1,526)=54.47, \mathrm{p}$-value $<0.001)$. As expected, the results reveal that the joystick was better than the Joyman in terms of speed of the navigation. The mean value for the completion time of a path was $187 \mathrm{~s}$ (standard deviation=13s) for the joystick and $321 \mathrm{~s}$ (standard deviation $=89 \mathrm{~s}$ ) for the Joyman.

A specific analysis was developed to study the learning effect of the two configurations (Joyman and Joystick). A linear model where all conditions are mixed was fitted to explain the relation between the task completion times and the trial number. For the Joyman configuration, it revealed that the slope of the linear regression was significantly lower than zero (p-value $<0.000001$ ), reflecting a significant decrease in the task completion time as the number of trials increases. The same analysis, where the first trial was removed, showed that the slope was not significantly different from zero anymore (for both visual conditions). Figure 8 illustrates the task completion time of the different trials for the Joyman configuration.

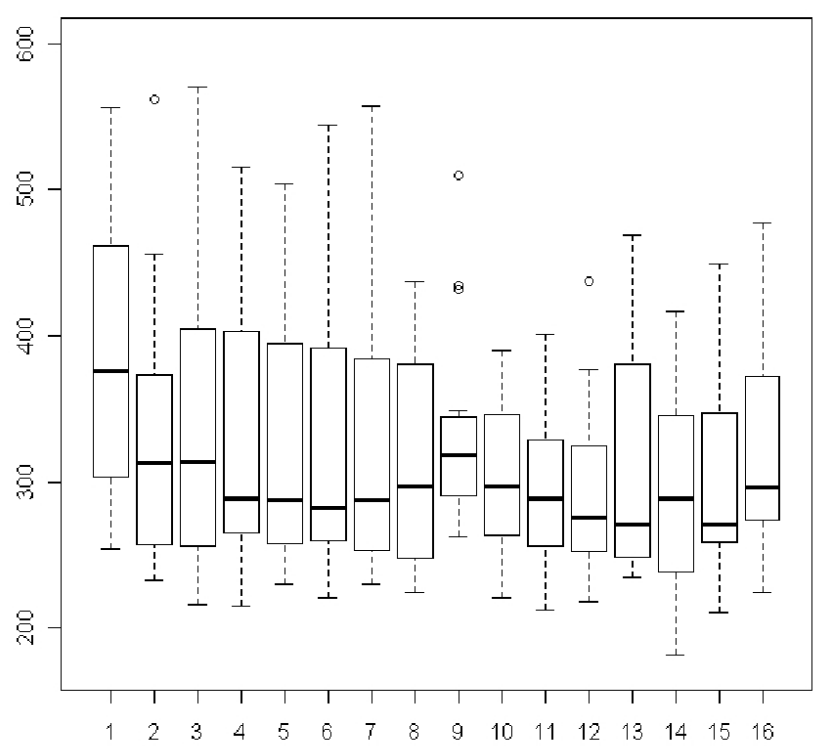

Figure 8: Task completion time (in seconds) for the Joyman configuration (16 trials). The first trial was left to illustrate the learning effect. Each boxplot is delimited by the quartile (25\% quantile and $75 \%$ quantile) of the distribution of the condition over the individuals. The median is also represented for each trial.

For each participant and for each trial, the percentage of errors for the different paths was measured. There was no error at the end, for both configurations (Joyman and Joystick) and both visual conditions.

\subsubsection{Subjective Questionnaire}

A preference questionnaire was proposed in which participants had to grade from 1 (low appreciation) to 7 (high appreciation) the two configurations (Joyman and Joystick) according to 8 subjective criteria : (a) Fun, (b) Intuitive, (c) Acccuracy, (d) Presence, (e) Rotation realism, (f) Fatigue, (g) Cybersickness and (i) Global appreciation. The grade 7 for Fatigue and Cybersickness respectively means that the interface does not induce any fatigue and does not imply any cybersickness feeling. Figure 9 shows the results concerning the grades (Likert-scale) obtained by the two different interfaces for each of the subjective criteria.

After performing an ANOVA on the two different conditions, we found a significant effect for 6 criteria: Fun $(F(1,30)=17.77$, $p<0.001)$, Intuitive $(F(1,30)=21.25$, p-value $<0.001)$, Accuracy $(F(1,30)=23.52$, p-value $<0.001)$, Presence $(F(1,30)=13.35, \mathrm{p}$ value $<0.001)$, Rotation realism $(F(1,30)=6.63$, p-value $=0.015)$ and Fatigue $(F(1,30)=87.51$, p-value $<0.001)$. In particular, our new platform was better ranked for Fun, Presence and Rotation realism. No significant effect was found for Cybersickness $(F(1,30)=4.01$, p-value $=0.054)$ and Global appreciation $(F(1,30)=0.69$, p-value $=0.411)$. 


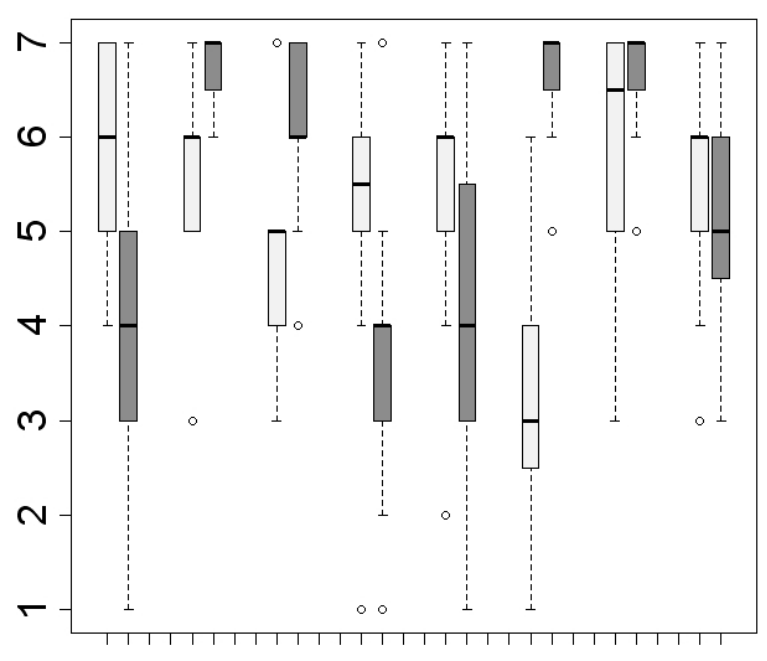

(a) (b) (c) (d) (e) (f) (g) (h)

Figure 9: Results for the subjective questionnaire for the two different configurations (Joyman and Joystick) with respect to a Likert-scale grading. The light color corresponds to the Joyman condition and the dark color corresponds to the joystick condition. The subjective criteria are (a) Fun, (b) Intuitive, (c) Accuracy, (d) Presence, (e) Rotation realism, (f) Fatigue, (g) Cybersickness (h) Global appreciation. Each boxplot is delimited by the quartile ( $25 \%$ quantile and $75 \%$ quantile) of the distribution of the condition over the individuals. The median is also represented for each condition.

\section{Discussion and Perspectives}

As a global conclusion of the preliminary evaluation of the Joyman, we can state that the feeling of immersion in the virtual world is significantly improved - in comparison with traditional joystick-based techniques - at the cost of some easiness of use. The Joyman is still at a early stage of development, however, first results are promising and open a large set of possible directions to improve usability and the level of realism of virtual navigation. This section discuss as exhaustively as possible these directions as well as future work to meet our objectives: an easy, intuitive, immersive interface allowing realistic locomotion in virtual worlds.

Interface Calibration The navigation with the Joyman implies the whole body and, as expected, the task completion times were higher for our interface compared to the joystick where only the arms and hands are involved. The use of the whole body, implying more movements, is also a reason for the lower rating for the Accuracy criterion in the subjective questionnaire. However, the results concerning the evaluation of our interface in terms of performances are encouraging as the participants always succeeded to complete the navigation task during the evaluation. One way of improvement for the performances of our interface could be the interface calibration. The control law directly transforms the angle of the interface platform into virtual walking velocities. 3 types of parameters were proposed to design our control law: those define the active angles area into the device state space $\left(\alpha_{x_{\text {min }}}, \alpha_{x_{\max }}, \alpha_{y_{\min }}\right.$ and $\alpha_{y_{\max }}, \alpha_{x_{0+}}, \alpha_{x_{0-}}, \alpha_{y_{0+}}$ and $\left.\alpha_{y_{0-}}\right)$, those controlling the reachable virtual velocities $\left(\dot{\theta}_{\max }, v_{t_{\min }}\right.$ and $\left.v_{t_{\max }}\right)$ and finally those controlling the dynamics of the relation between tangential and angular velocities (a, b, and c, see Equation 3). Ideally, the first type of parameters should be calibrated for each user. Indeed, given his size, weight and strength, each user provides relatively different efforts to reach a same given platform inclination angle (i.e., a given walking velocity). The slight fatigue reported by participants in the results of our experiments confirms the need for individual calibration. Currently, calibration process consists in recording leaning motions as well as neutral positions to define the bounds of the effective zone for the platform. We made such a calibration before experiments, but kept a unique one all along our evaluation in order to have the same behavior of our interface for all participants. For VR applications, we could envisage to explore various calibration techniques as well, such as by displaying a virtual environment with a moving point-of-view and to ask users to apply the effort on the platform they believe to be corresponding to the motion.

Mechanical design We presented in this paper the first prototype of our new interface. The results of the subjective questionaire suggest that the participants enjoyed the navigation with the Joyman. They namely gave a higher rating for the Fun criterion but also for Presence criterion and Rotation Realism criterion. Specifically, the higher rate given to this last criterion confirms that our mechanical design was appreciated for locomotion tasks in the virtual environment. A way of improvement for our mechanical design could concern the linkage between the platform and the basis. The proposed prototype implements this linkage using an inextensible rope and a set of springs (see Figure 4) as we wanted to have a simple mechanical design. However, we could envisage to modify the linkage to increase the possibility of movements of our interface. Ideally (mechanically speaking), the linkage could be a 2 rotational degrees of freedom, one with a restoring force proportional to the platform inclination.

Control Law Experimental evaluation reveals that the proposed control law is intuitively grasped by users: establishing a relation between linear and angular velocities seems to be naturally accepted by users, and is consistent with observations of human locomotion trajectories. Future work will deal with potential modifications of the control law, for example by using a law that can lower vestibular and visual sensory conflicts.

The device state vector is currently two dimensional: the two angles that describe the platform inclination. With some experience, it appears that the device can be fully and accurately controlled by involving the lower body only, it is not even required to hang on to the barrier (except for moving backward in the current state of the device). Such a property opens interesting perspectives and makes possible to increase the dimension of the device state space. Possible extensions are numerous, and immediately within reach if the VR system is equipped with tracking abilities: hands remain free to achieve secondary actions in the virtual worlds (grasping, touching, pointing tasks, etc.), view direction and locomotion control can be decomposed, etc. Nevertheless, we observed during experiments that most of the participants intuitively attempts to control their locomotion also by moving their upper body as illustrated in Figure 10, in spite of the inefficiency of such motions to significantly increase the inclination of the platform. We however could use this input (i.e., the orientation of the spine relatively to the hips) to control navigation based on an holonomic locomotion model: as opposed to the non-holonomic one, lateral velocities are allowed in addition to tangential and angular ones by removing the constraint imposed by equation 2 (lateral velocities here correspond to sidesteps).

Immersion and sensory feedback Evaluation showed that the Joyman provides a satisfying level of immersion into the virtual world. In addition, the Presence criterion was better ranked for our interface, compared to the joystick. As futur work, we could envisage to improve the level of immersion by adding other interaction techniques. Thus, the visual perception of motion could be improved by adding camera motions like in [16] for example. Thus, 


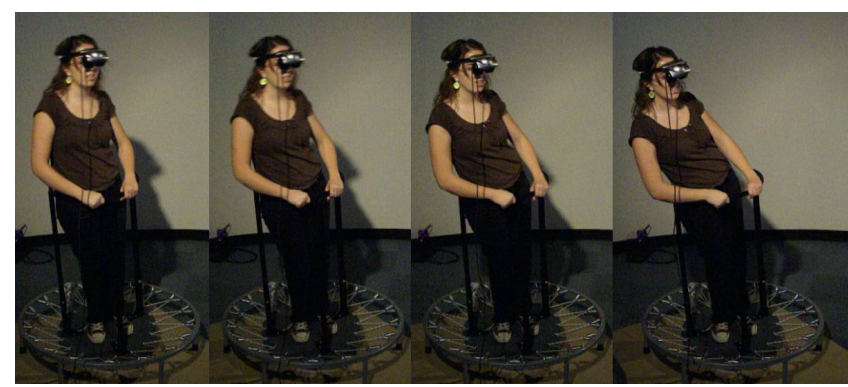

Figure 10: 4 Snapshots extracted from a video sequence acquired during evaluation experiments. We could observe that participants intuitively attempt to use their upper body to control their virtual locomotion in spite of the inefficiency of such actions (note that the platform inclination is almost unchanged from picture to picture). However, this observation provides interesting direction to improve virtual motion controllability, and eventually control side steps from such inputs.

having oscillating view point could reinforce the accuracy of the perception of the traveled distance by reproducing the natural oscillations of the head during human locomotion. This could also lower the feeling that locomotion is too slow as reported by participants in the subjective questionnaire.

\section{Conclusion}

In this paper, we presented a novel interface to control locomotion into a virtual world while remaining globally static in the real space. The Joyman interface is composed of a new peripheral device and a dedicated control law which transforms the device state into a virtual locomotion velocity vector. Our main contributions are (1) to explore the users ability to exploit equilibrioception to control their virtual locomotion and (2) to maintain a high level of immersion compared to handheld devices (e.g., joysticks).

After a preliminary evaluation, we obtained promising results as the users enjoyed the navigation with our new interface. Presence and realism in the virtual rotations were also underlined. The evaluation also opened several future work directions to improve and extend our interface. Various VR but also real applications could be envisaged, when navigating in a 3D world. Our interface could be used for example for videogames, rehabilitation, training tasks or virtual visits.

A patent for the interface presented in this paper has been filed on November 19, 2010 under the number FR10/595551.

\section{REFERENCES}

[1] G. Arechavaleta, J.-P. Laumond, H. Hicheur, and A. Berthoz. The nonholonomic nature of human locomotion: a modeling study. In Proceedings of IEEE International Conference on Biomedical Robotics and Biomechatronics, pages 158-163, 2006.

[2] S. Beckhaus, K. Blom, and M. Haringer. ChairIO - the Chair-Based Interface. Ed. Magerkurth und Rötzler, Shaker Verlag, 2007.

[3] D. Bowman, E. Kruijff, J. LaViola, and I. Poupyrev. 3D User Interfaces: Theory and Practice. Addison-Wesley Boston (MA), 2005.

[4] G. Cirio, M. Marchal, T. Regia-Corte, and A. Lécuyer. The magic barrier tape: a novel metaphor for infinite navigation in virtual worlds with a restricted walking workspace. In Proceedings of the ACM Symposium on VRST, pages 155-162, 2009.

[5] K. Fernandes, V. Raja, and J. Eyre. Cybersphere: the fully immersive spherical projection system. Communications of the ACM, 46(9):146, 2003.

[6] H. Iwata, H. Yano, H. Fukushima, and H. Noma. Circulafloor: A locomotion interface using circulation of movable tiles. In Proceedings of IEEE Virtual Reality Conference, pages 223-230, 2005.
[7] H. Iwata, H. Yano, and F. Nakaizumi. Gait master: A versatile locomotion interface for uneven virtual terrain. In Proceedings of IEEE Virtual Reality Conference, pages 131-137, 2001.

[8] H. Iwata and Y. Yoshida. Path reproduction tests using a torus treadmill. Presence: Teleoperators and Virtual Environments, 8(6):587597,1999

[9] A. Lécuyer, J.-M. Burkhardt, J.-M. Henaff, and S. Donikian. Camera motions improve the sensation of walking in virtual environments. In Proceedings of IEEE Virtual Reality Conference, pages 11-18, 2006.

[10] N. Nitzsche, U. Hanebeck, and G. Schmidt. Motion compression for telepresent walking in large target environments. Presence: Teleoperators and Virtual Environments, 13(1):44-60, 2004.

[11] R. Pausch, T. Burnette, D. Brockway, and M. E. Weiblen. Navigation and locomotion in virtual worlds via flight into hand-held miniatures. In Proceedings of SIGGRAPH'95, pages 399-400, 1995.

[12] S. Razzaque, Z. Kohn, and M. C. Whitton. Redirected walking. In Proceedings of Eurographics, 2001.

[13] M. Schwaiger, T. Thummel, and H. Ulbricht. A 2d-motion platform: The cybercarpet. In Proceedings of Second Joint Eurohaptics Conference and Symposium on Haptic Interfaces for Virtual Environment and Teleoperator Systems, pages 415-420, 2007.

[14] M. Slater, M. Usoh, and A. Steed. Taking steps: The influence of a walking technique on presence in virtual reality. ACM Transactions on Computer-Human Interaction, 2(3):201-219, 1995.

[15] K. Stanney and al. Handbook of Virtual Environments: Design, Implementation, and Applications. Lawrence Erlbaum Associates, 2002.

[16] L. Terziman, A. Lécuyer, S. Hillaire, and J. M. Wiener. Can camera motions improve the perception of traveled distance in virtual environments? In Proceedings of IEEE Virtual Reality Conference, pages 131-134, 2009.

[17] J. Torchinsky. Giant atari 2600 joystick. http://www.vgg. com/jason/jdt/projects.html, 2006. [Online; accessed 6January-2011].

[18] B. Williams, G. Narasimham, B. Rump, T. McNamara, T. Carr, J. Rieser, and B. Bodenheimer. Exploring large virtual environments with an hmd when physical space is limited. In Proceedings of the ACM Symposium on Applied Perception in Graphics and Visualization, pages 41-48, 2007.

[19] Y. Zhang, J. Pettré, Q. Peng, and S. Donikian. Data based steering of virtual human using a velocity-space approach. In Proceedings of Motion in Games, Lecture Notes in Computer Science 5884, pages 170-181. Springer, 2009. 